\title{
Dynamical Casimir Effect in a one-dimensional uniformly contracting cavity
}

\author{
A.M. Fedotov, Yu.E. Lozovik ${ }^{\dagger}$, N.B. Narozhny, and A.N. Petrosyan \\ Moscow Engineering Physics Institute, 115409 Moscow, Russia and \\ ${ }^{\dagger}$ Institute of Spectroscopy of Russian Academy of Science, Troitsk, 142190 Moscow region, Russia
}

\begin{abstract}
We consider particle creation (the Dynamical Casimir effect) in a uniformly contracting ideal one-dimensional cavity non-perturbatively. The exact expression for the energy spectrum of created particles is obtained and its dependence on parameters of the problem is discussed. Unexpectedly, the number of created particles depends on the duration of the cavity contracting non-monotonously. This is explained by quantum interference of the events of particle creation which are taking place only at the moments of acceleration and deceleration of a boundary, while stable particle states exist (and thus no particles are created) at the time of contracting.
\end{abstract}

PACS numbers: 42.50.Ct,42.50.Dv,03.65.-w

\section{INTRODUCTION}

During the last two decades the dynamical Casimir effect (DCE), the effect of photon creation in an empty nonstationary cavity [1], attracted considerable attention in literature. Being a firm prediction of quantum field theory, the DCE has not been observed yet experimentally. This is probably the main reason for such interest to it, and therefore search for a realistic scheme for experimental observation of the DCE is an actual problem of modern quantum electrodynamics.

A nonstationary cavity can be realized by two possible ways: (i) a cavity with moving boundaries, or (ii) a cavity with fixed shape but with varying boundary conditions. In case (i) a significant number of photons could be created if the velocity of boundaries is close to the speed of light. The corresponding experimental realization would be obviously a difficult technical problem. Alternatively, it was suggested to utilize the vibrating cavity and accumulate the effect by tuning the frequency of small mechanical oscillations of the boundaries (which could be induced, e.g., by a sound wave [2], or due to piezoelectric effect [3]) in resonance with an eigenfrequency of the cavity [4, [5, [6, 7, []], see also the recent review [9]. In this realization, an extremely high accuracy of frequency tuning must be provided.

Owing to amazing progress in modern laser technology and semiconductor electronics, the variant (ii) could be considered as a more realistic one [10, 11, 12]. The optical properties of a semiconductor film located on a dielectric base can be changed, e.g., by means of electron-hole plasma creation by a strong femtosecond laser pulse [1]], or due to injection of carriers by a powerful electric pulse [13]. In both cases, variation of optical properties of the boundary can be fast enough and well controlled.

However, theoretical investigation of the first variant is simpler from mathematical point of view, at least in the framework of a one-dimensional model. Sometimes in the framework of the first approach exact solutions to the problem can be found for some special laws of motion. Besides, one may hope that very fast displacement of a boundary acts like "almost instant" creation of a new boundary due to changing of optical properties of the medium inside the cavity. Therefore we will consider a cavity with moving boundaries in this paper, and will be especially interested in the case of ultrarelativistic motion.

We will present a new exact solution for the DCE problem in a one-dimensional ideal cavity uniformly contracting 
during the time interval $0<t<T$ and otherwise stationary. A complete set of solutions for a classical field inside a one- or even three-dimensional cavity at the time of uniform contracting was known for years, see Ref. [9]. It was used, for example, in Ref. 14, 15, for calculation of the Casimir force acting between the boundaries of a relativistically squeezing or expanding cavity. In contrast, our solution for a one-dimensional quantum field in a uniformly contracting cavity seems to be unknown in literature, and allows one to study the DCE for this particular case non-perturbativly. We have discovered a new and unexpected effect of periodic dependence of the number of created particles on some variable $\chi_{f}$ determined by the time of contracting $T$ and velocity of the boundary $v$. It is explained by quantum interference of the events of particle creation which are taking place only at the moments of acceleration $(t=0)$ and deceleration $(t=T)$ of a boundary, while stable particle states exist (and thus no particles are created) at the time of contracting.

We review the Hamiltonian approach in Section III Consideration of a field quantization procedure in a uniformly contracting cavity is presented in Section III Section IV is devoted to calculation of the energy spectrum of particles created in the cavity. The periodic dependence of all measurable quantities on $\chi_{f}$ is indicated, and the optimal conditions for the DCE are derived. The discussion of the results and conclusions are given in Section $\nabla$ We use natural units $\hbar=c=1$ throughout the paper.

\section{HAMILTONIAN AND OTHER PRELIMINARIES}

We will use the Hamiltonian approach which in application to DCE was apparently first formulated in Ref. [16], see also [6, [9]. In this section we begin with derivation of Hamiltonian for a one-dimensional problem based on the well known properties of time-dependent canonical transformations in classical Hamiltonian mechanics (see, e.g., 17]). Our approach clears up the presence of extra terms in the Law Hamiltonian and is equivalent to the method developed in the Law's paper [6], at least, for quantum systems with quadratic Hamiltonian.

Consider a massless scalar quantum field $\Phi(x, t)$ in a nonstationary one-dimensional cavity formed by two mirrors. One of the mirrors is fixed at the point $x=0$, while the position of the other depends on time $x=l(t)$. For definiteness, we impose the following boundary conditions at the mirrors: $\Phi(0, t)=\Phi(l(t), t)=0$.

The Hamiltonian for the field reads

$$
H=\frac{1}{2} \int_{0}^{l(t)}\left\{\left(\frac{\partial \Phi(x, t)}{\partial t}\right)^{2}+\left(\frac{\partial \Phi(x, t)}{\partial x}\right)^{2}\right\} d x .
$$

The field $\Phi(x, t)$ and the canonical momentum $\Pi(x, t)=\partial \Phi(x, t) / \partial t$ are assumed to obey the equal time commutation relations $\left[\Phi(x, t), \Pi\left(x^{\prime}, t\right)\right]=i \delta\left(x-x^{\prime}\right)$, and hence the Heisenberg equations acquire the form

$$
\dot{\Phi}(x, t)=i[H, \Phi(x, t)]=\Pi(x, t), \quad \dot{\Pi}(x, t)=i[H, \Pi(x, t)]=\frac{\partial^{2} \Phi(x, t)}{\partial x^{2}} .
$$

One can easily see that the Fourier coefficients

$$
\begin{aligned}
Q_{n}(t) & =\sqrt{\frac{2}{l(t)}} \int_{0}^{l(t)} \Phi(x, t) \sin \left(\frac{\pi n x}{l(t)}\right) d x, \\
P_{n}(t) & =\sqrt{\frac{2}{l(t)}} \int_{0}^{l(t)} \Pi(x, t) \sin \left(\frac{\pi n x}{l(t)}\right) d x,
\end{aligned}
$$

satisfy the standard commutation relations $\left[Q_{n}, P_{n^{\prime}}\right]=i \delta_{n n^{\prime}}$ and $\left[Q_{n}, Q_{n^{\prime}}\right]=\left[P_{n}, P_{n^{\prime}}\right]=0$. Introducing the "instantaneous eigenfrequencies" $\omega_{n}(t)=\pi n / l(t)$, and the "instantaneous destruction and creation" operators $a_{n}, a_{n}^{\dagger}$

$$
a_{n}(t)=\frac{\omega_{n}(t) Q_{n}(t)+i P_{n}(t)}{\sqrt{2 \omega_{n}(t)}}, \quad a_{n}^{\dagger}(t)=\frac{\omega_{n}(t) Q_{n}(t)-i P_{n}(t)}{\sqrt{2 \omega_{n}(t)}}
$$

which satisfy the canonical commutation relations $\left[a_{n}, a_{n^{\prime}}^{\dagger}\right]=\delta_{n n^{\prime}},\left[a_{n}, a_{n^{\prime}}\right]=\left[a_{n}^{\dagger}, a_{n^{\prime}}^{\dagger}\right]=0$, one can write the 
expansions for the field and canonical momentum in the form

$$
\begin{gathered}
\Phi(x, t)=\sqrt{\frac{2}{l(t)} \sum_{n} \sin \left(\frac{\pi n x}{l(t)}\right) \frac{a_{n}(t)+a_{n}^{\dagger}(t)}{\sqrt{2 \omega_{n}(t)}},} \\
\Pi(x, t)=\sqrt{\frac{2}{l(t)}} \sum_{n} \sin \left(\frac{\pi n x}{l(t)}\right)\left(-i \sqrt{\frac{\omega_{n}(t)}{2}}\right)\left\{a_{n}(t)-a_{n}^{\dagger}(t)\right\} .
\end{gathered}
$$

Since operators $a_{n}, i a_{n}^{\dagger}$ and $Q_{n}, P_{n}$ satisfy the same commutation relations, Eqs. (6) can be considered as a canonical transformation. One can easily check that this transformation can be defined with a generating functional $F[a, \Phi]$ as

$$
\begin{gathered}
a_{n}^{\dagger}=-i \frac{\partial F[a, \Phi]}{\partial a_{n}}, \quad \Pi(x, t)=-\frac{\delta F[a, \Phi]}{\delta \Phi(x, t)}, \\
F[a, \Phi]=-\frac{i}{2} \sum_{n}\left\{\omega_{n}(t) Q_{n}^{2}[\Phi, t]+a_{n}^{2}-2 \sqrt{2 \omega_{n}(t)} Q_{n}[\Phi, t] a_{n}\right\} .
\end{gathered}
$$

The order ambiguity in the last term of Eq. (8) can give rise only to an additive indefinite c-number constant, and thus is insignificant.

For the stationary case $(l(t)=$ const) the generating functional does not depend on time explicitly, so that the Hamiltonian remains unchanged under the canonical transformation (7), (8). In terms of new variables it acquires the form $H=\sum_{n} \omega_{n} a_{n}^{\dagger} a_{n}$. If the cavity is nonstationary, and hence the generating functional explicitly depends on time, the Hamiltonian changes, compare [17]. The operators $a_{n}$ satisfy the equations $\dot{a}_{n}=i\left[H^{\prime}, a_{n}\right]$, where the new Hamiltonian is $H^{\prime}=H-\partial F / \partial t$.

For the case of a uniformly contracting cavity

$$
l(t)= \begin{cases}l_{i}, & t<0 \\ l_{i}-v t, & 0<t<T \\ l_{f}, & t>T\end{cases}
$$

where $T=\left(l_{f}-l_{i}\right) / v$ is duration of contracting and $v>0$ is the velocity of the moving boundary, we obtain at $0<t<T$

$$
H^{\prime}=\pi \Lambda / l(t), \quad \Lambda=\sum_{n}\left\{n a_{n}^{\dagger} a_{n}-i \frac{v}{4 \pi}\left(a_{n}^{2}-a_{n}^{\dagger}{ }^{2}\right)\right\}-\frac{i v}{\pi} \sum_{n \neq n^{\prime}}(-1)^{n+n^{\prime}} \sqrt{n n^{\prime}} \times\left\{\frac{\left(a_{n} a_{n^{\prime}}-a_{n^{\prime}}^{\dagger} a_{n}^{\dagger}\right)}{2\left(n+n^{\prime}\right)}+\frac{a_{n}^{\dagger} a_{n^{\prime}}}{n^{\prime}-n}\right\} .
$$

The same result follows from the general Law formula (2.21) of Ref. [6] for the "effective" Hamiltonian which was constructed to reduce the equations of motion for operators $Q_{n}, P_{n}$ to the Heisenberg form. This proves that both approaches are equivalent at least for the case when the Hamiltonian is quadratic in structure.

The additional terms in (10) are responsible for annihilation and creation of pairs and scattering of particles from one mode to another in the contracting cavity. The corresponding "coupling constants" are proportional to the velocity of the moving boundary and are small in non-relativistic case. One can see that the terms of the Hamiltonian (10) responsible for interaction between modes are of the same order as those describing particle production in a separate mode. Therefore strong interaction between field modes is a built-in internal feature of DCE [18].

We see from Eq. (10) that the Hamiltonian $H^{\prime}$ has a remarkable property. Its time dependence is determined exclusively by a c-number factor $\pi / l(t)$. Hence, the operator $\Lambda$ does not depend on time explicitly and is an integral of motion. Therefore, being quadratic in canonical variables $a_{n}, a_{n}^{\dagger}$, it admits diagonalization by a time independent linear canonical transformation (Bogolubov transformation) from $a_{n}, a_{n}^{\dagger}$ to some new canonical variables $\tilde{b}_{n}, \tilde{b}_{n}^{\dagger}$. In terms of these new variables we would have

$$
\Lambda=\sum_{n} \lambda_{n} \tilde{b}_{n}^{\dagger} \tilde{b}_{n}, \quad H^{\prime}=\sum_{n}\left(\pi \lambda_{n} / l(t)\right) \tilde{b}_{n}^{\dagger} \tilde{b}_{n}
$$

Hereinafter it is convenient to introduce an auxiliary dimensionless time variable $\chi=\pi \int_{0}^{t} d t / l(t)$, which varies from $-\infty$ to $+\infty$. At $0<t<T, \chi=-(\pi / v) \ln \left(1-v t / l_{i}\right)$. In terms of this variable the Heisenberg equations of motion acquire the form

$$
d a_{n} / d \chi=i\left[\Lambda, a_{n}\right], \quad 0<t<T,
$$

so that the operator $\Lambda$ can be considered as the generator of translations in "time" $\chi$. The time moment $t=0$ corresponds to $\chi_{i}=0$, whereas $t=T$ to $\chi_{f}=(\pi / v) \ln (1 / \rho)$, where the dimensionless parameter $\rho=l_{f} / l_{i}<1$ is the squeeze rate for the cavity. The value $\chi=\infty$ corresponds to the moment $t=l_{i} / v$ of the cavity collapse. 


\section{DIAGONALIZATION OF THE HAMILTONIAN}

We will use the so-called Milne reference frame, see, e.g., Ref. [19], to diagonalize operator $\Lambda$. The corresponding spatial $\xi$ and time $\tau$ Milne coordinates are defined by

$$
t=\frac{l_{i}}{v}-\tau \cosh \xi, \quad x=\tau \sinh \xi,
$$

The point is that the cavity remains stationary in Milne reference frame. Indeed, the spacetime region $0<x<l_{i}-v t$ is mapped conformally by transformation (13) onto the strip $0<\xi<(1 / 2) \ln d, \tau>0$, where $d=(1+v) /(1-v)$ coincides with the Doppler factor for reflection from a mirror moving with velocity $v$. This means that the world line for the right boundary is given by $\xi=(1 / 2) \ln d=$ const.

The wave equation in terms of Milne coordinates reads:

$$
\frac{1}{\tau} \frac{\partial}{\partial \tau}\left(\tau \frac{\partial \Phi}{\partial \tau}\right)-\frac{1}{\tau^{2}} \frac{\partial^{2} \Phi}{\partial \xi^{2}}=0
$$

This equation can be easily solved by separation of variables. Taking into account boundary conditions (which are stationary in the Milne reference frame) we obtain a complete set of modes

$$
\Psi_{n}(\xi, \tau)=\frac{1}{\sqrt{\pi n}}\left(\frac{v \tau}{l_{i}}\right)^{2 \pi i n / \ln d} \sin \left(\frac{2 \pi n \xi}{\ln d}\right), \quad n=1,2, \ldots,
$$

which are normalized by the relation

$$
-i \int_{0}^{\frac{1}{2} \ln d} d \xi \tau\left[\Psi_{n}^{*} \frac{\stackrel{\leftrightarrow}{\partial}}{\partial \tau} \Psi_{n^{\prime}}\right]=\delta_{n n^{\prime}}
$$

Note that the unusual sign in the LHS of this equation arises due to opposite directions of physical time $t$ and Milne time $\tau$. In terms of the original variables $x, t$ these modes acquire the form

$$
\Psi_{n}(x, t)=\frac{-i}{2 \sqrt{\pi n}}\left\{\left(1-\frac{v(t-x)}{l_{i}}\right)^{\frac{2 \pi i n}{\ln d}}-\left(1-\frac{v(t+x)}{l_{i}}\right)^{\frac{2 \pi i n}{\ln d}}\right\},
$$

and constitute a complete set of solutions for the wave equation in a uniformly contracting cavity orthonormalized by the usual Klein-Gordon scalar product

$$
i \int_{0}^{l(t)} d x\left[\Psi_{n}^{*} \frac{\stackrel{\leftrightarrow}{\partial}}{\partial t} \Psi_{n^{\prime}}\right]=\delta_{n n^{\prime}}
$$

Hence, the general solution to the Heisenberg equations (2) can be represented in the form

$$
\Phi(x, t)=\sum_{n}\left\{b_{n} \Psi_{n}(x, t)+b_{n}^{\dagger} \Psi_{n}^{*}(x, t)\right\},
$$

where operators $b_{n}, b_{n}^{\dagger}$ satisfy the standard Bose-Einstein commutation relations and are independent of time since modes (16) satisfy the wave equation exactly. Specifically, this means that the Hamiltonian which governs behavior of the variables $b_{n}$ and $b_{n}^{\dagger}$ is equal to zero with an accuracy to a $c$-number contribution.

Let us now express operators $b_{n}$ in terms of operators $a_{n}, a_{n}^{\dagger}$. Due to completeness and orthonormality of the set (16), we have:

$$
b_{n}=i \int_{0}^{l(t)}\left\{\Psi_{n}^{*}(x, t) \Pi(x, t)-\frac{\partial \Psi_{n}^{*}(x, t)}{\partial t} \Phi(x, t)\right\} d x .
$$

Using expansions (6) we reduce RHS of Eq. (18) to the form

$$
b_{n}=\sum_{n^{\prime}}\left\{A_{n n^{\prime}}(t) a_{n^{\prime}}(t)+B_{n n^{\prime}}(t) a_{n^{\prime}}^{\dagger}(t)\right\},
$$


where

$$
\left.\begin{array}{l}
A_{n n^{\prime}}(t) \\
B_{n n^{\prime}}(t)
\end{array}\right\}=\frac{-i}{\sqrt{\pi n^{\prime}}} \int_{0}^{l(t)} d x \sin \left(\frac{\pi n^{\prime} x}{l(t)}\right)\left\{\frac{\partial \Psi_{n}^{*}(x, t)}{\partial t} \pm i \omega_{n^{\prime}} \Psi_{n}^{*}(x, t)\right\} .
$$

Now, using explicit expressions (16) we can express time derivatives in Eq. (19) in terms of derivatives with respect to $x$ and perform integration by parts. Introducing then a scaling variable $y=x / l(t)$ we finally obtain:

$$
A_{n n^{\prime}}(t)=e^{2 i v n \chi(t) / \ln d} \alpha_{n n^{\prime}}, \quad B_{n n^{\prime}}(t)=e^{2 i v n \chi(t) / \ln d} \beta_{n n^{\prime}},
$$

where coefficients

$$
\left.\begin{array}{l}
\alpha_{n n^{\prime}} \\
\beta_{n n^{\prime}}
\end{array}\right\}=\frac{1}{2} \sqrt{\frac{n^{\prime}}{n}} \int_{-1}^{1} d y(1-v y)^{-2 \pi i n / \ln d} e^{\mp i \pi n^{\prime} y}
$$

are already independent of time $t$, or auxiliary variable $\chi$.

Thus, we have

$$
b_{n}=e^{2 i v n \chi(t) / \ln d} \tilde{b}_{n}(t),
$$

where operators

$$
\tilde{b}_{n}(t)=\sum_{n^{\prime}}\left\{\alpha_{n n^{\prime}} a_{n^{\prime}}(t)+\beta_{n n^{\prime}} a_{n^{\prime}}^{\dagger}(t)\right\},
$$

are expressed in terms of operators $a_{n}, a_{n}^{\dagger}$ by a Bogolubov transformation with coefficients, which do not depend on time explicitly. Therefore the Hamiltonian which determines time dependence of the operators $\tilde{b}_{n}$ remains unchanged under transformation (23) and is given by Eq. (10). This result, together with Eq. (22), is sufficient to ascertain the structure of the Hamiltonian $\Lambda$ in terms of the operators $\tilde{b}_{n}$.

Indeed, the Heisenberg equations of motion for operators $\tilde{b}_{n}$ are given by $d \tilde{b}_{n} / d \chi=i\left[\Lambda, \tilde{b}_{n}\right]$, compare (12). Taking into account the form (22) of time dependence of $\tilde{b}_{n}$, we have for the commutator $\left[\Lambda, \tilde{b}_{n}\right]$ :

$$
\left[\Lambda, \tilde{b}_{n}\right]=-(2 v / \ln d) n \tilde{b}_{n} .
$$

It clearly follows from (10) and (23) that $\Lambda$ is quadratic in terms of operators $\tilde{b}_{n}$ also. Hence, we unambiguously arrive to

$$
\Lambda=\frac{2 v}{\ln d} \sum_{n} n \tilde{b}_{n}^{\dagger} \tilde{b}_{n}
$$

Eqs. (24), (23) and (21) constitute an exact solution for quantum field in a uniformly contracting cavity. This is one of the primary results of the present paper. Its physical meaning consists in existence of a notion of stable, non-interacting particles in a uniformly contracting one-dimensional cavity. Since pair production or mode-to-mode scattering are absent in this representation, the number of these particles in every mode is fixed. Their energies, however, depend on time due to collisions with the moving boundary, compare Eq. (11). It is worth noting that the exact energy spectrum of these particles turns out to be equidistant.

It is important that the operators $\tilde{b}_{n}(t)$ for the case of a stationary cavity coincide with $a_{n}(t)$. Indeed, proceeding to the limit $v \rightarrow 0$ in Eq. (21), one can easily make certain that $\alpha_{n, n^{\prime}}(0)=\delta_{n n^{\prime}}, \beta_{n, n^{\prime}}(0)=0$. We may conclude that operators $\tilde{b}_{n}(t), \tilde{b}_{n}^{\dagger}(t)$ defined by Eqs. (23),(21) represent a natural generalization for particle destruction and creation operators valid for nonstationary cavities with uniformly moving boundaries.

Let us now consider the Bogolubov transformation (23) with coefficients $\alpha_{n, n^{\prime}}(v), \beta_{n, n^{\prime}}(v)$ defined by Eq. (21) for $v(t)$ being an arbitrary function of time. Since coefficients of the transformation (23) now depend on time, the operators $\tilde{b}_{n}(t)$ are ruled by a new Hamiltonian $\widetilde{H}$, so that the Heisenberg equations of motion acquire the form $\dot{\vec{i}}_{n}=\left[\widetilde{H}, \widetilde{b}_{n}\right]$. Using Eqs. (23) and equations of motion for operators $a_{n}$, we obtain for the time derivative of $\tilde{b}_{n}(t)$ :

$$
\begin{aligned}
& \dot{\widetilde{b}}_{n}=\sum_{n^{\prime}}\left(\dot{\alpha}_{n n^{\prime}} a_{n^{\prime}}+\dot{\beta}_{n n^{\prime}} a_{n^{\prime}}^{\dagger}+\alpha_{n n^{\prime}} \dot{a}_{n^{\prime}}+\beta_{n n^{\prime}} \dot{a}_{n^{\prime}}^{\dagger}\right)= \\
& =\sum_{n^{\prime}}\left(\dot{\alpha}_{n n^{\prime}} a_{n^{\prime}}+\dot{\beta}_{n n^{\prime}} a_{n^{\prime}}^{\dagger}+\alpha_{n n^{\prime}} i\left[H^{\prime}, a_{n^{\prime}}\right]+\beta_{n n^{\prime}} i\left[H^{\prime}, a_{n^{\prime}}^{\dagger}\right]\right)=\sum_{n^{\prime}}\left(\dot{\alpha}_{n n^{\prime}} a_{n^{\prime}}+\dot{\beta}_{n n^{\prime}} a_{n^{\prime}}^{\dagger}\right)+i\left[H^{\prime} \widetilde{b}_{n}\right] .
\end{aligned}
$$


We know already the structure of $H^{\prime}$ in terms of $\widetilde{b}_{n}$, see Eqs. (10) and (24). For the difference $\widetilde{H}-H^{\prime} \equiv \mathcal{V}$ we have:

$$
i\left[\mathcal{V}, \widetilde{b}_{n}\right]=\sum_{n^{\prime}}\left(\dot{\alpha}_{n n^{\prime}} a_{n^{\prime}}+\dot{\beta}_{n n^{\prime}} a_{n^{\prime}}^{\dagger}\right) .
$$

Using the well known unitarity relations for Bogolubov coefficients

$$
\sum_{n^{\prime \prime}}\left(\alpha_{n n^{\prime \prime}} \beta_{n^{\prime} n^{\prime \prime}}-\alpha_{n^{\prime} n^{\prime \prime}} \beta_{n n^{\prime \prime}}\right)=0, \quad \sum_{n^{\prime \prime}}\left(\alpha_{n n^{\prime \prime}} \alpha_{n^{\prime} n^{\prime \prime}}^{*}-\beta_{n n^{\prime \prime}} \beta_{n^{\prime} n^{\prime \prime}}^{*}\right)=\delta_{n n^{\prime}},
$$

which in our case (21) can be verified straightforwardly, we get the inverse transformation

$$
a_{n}=\sum_{n^{\prime}}\left(\alpha_{n^{\prime} n}^{*} \widetilde{b}_{n^{\prime}}-\beta_{n^{\prime} n} \widetilde{b}_{n^{\prime}}^{\dagger}\right)
$$

and hence,

$$
i\left[\mathcal{V}, \widetilde{b}_{n}\right]=\dot{v} \sum_{n^{\prime}}\left(\mathcal{A}_{n n^{\prime}} \widetilde{b}_{n^{\prime}}+\mathcal{B}_{n n^{\prime}} \widetilde{b}_{n^{\prime}}^{\dagger}\right)
$$

where

$$
\mathcal{A}_{n n^{\prime}}=\sum_{n^{\prime \prime}}\left(\frac{d \alpha_{n n^{\prime \prime}}}{d v} \alpha_{n^{\prime} n^{\prime \prime}}^{*}-\beta_{n^{\prime} n^{\prime \prime}}^{*} \frac{d \beta_{n n^{\prime \prime}}}{d v}\right), \quad \mathcal{B}_{n n^{\prime}}=\sum_{n^{\prime \prime}}\left(\frac{d \beta_{n n^{\prime \prime}}}{d v} \alpha_{n^{\prime} n^{\prime \prime}}-\beta_{n^{\prime} n^{\prime \prime}} \frac{d \alpha_{n n^{\prime \prime}}}{d v}\right) .
$$

Substituting representations (21) for Bogolubov coefficients into Eqs. (28) we can perform summations over $n^{\prime \prime}$ in (28) using the well known relation

$$
\sum_{n=-\infty}^{\infty} n e^{i \pi x n}=-\frac{2 i}{\pi} \sum_{k=-\infty}^{\infty} \delta^{\prime}(x-2 k)
$$

After this, all integrations become trivial and we obtain:

$$
\begin{gathered}
\mathcal{A}_{n n^{\prime}}=i \frac{(-1)^{n^{\prime}-n}}{\pi} \frac{\sqrt{n n^{\prime}} \gamma^{2+2 i \pi\left(n^{\prime}-n\right) / \ln d}}{\left(n^{\prime}-n\right)\left(n^{\prime}-n+i \ln d / 2 \pi\right)}, \quad n \neq n^{\prime}, \quad \mathcal{A}_{n n}=-\frac{2 i \pi n}{\ln ^{2} d}\left[\frac{\ln d}{v}+2 \gamma^{2}(\ln \gamma)-1\right], \\
\mathcal{B}_{n n^{\prime}}=i \frac{(-1)^{n^{\prime}+n}}{\pi} \frac{\sqrt{n n^{\prime}} \gamma^{2-2 i \pi\left(n^{\prime}+n\right) / \ln d}}{\left(n^{\prime}+n\right)\left(n^{\prime}+n-i \ln d / 2 \pi\right)},
\end{gathered}
$$

where $\gamma=\left(1-v^{2}\right)^{-1 / 2}$.

It follows from (29) that $\mathcal{A}_{n n^{\prime}}^{*}=-\mathcal{A}_{n^{\prime} n}, \mathcal{B}_{n n^{\prime}}=\mathcal{B}_{n^{\prime} n}$. Using these relations and Eq. (27) one can easily find

$$
\mathcal{V}=i \dot{v}(t) \sum_{n, n^{\prime}}\left\{\mathcal{A}_{n n^{\prime}}(v(t)) \widetilde{b}_{n}^{\dagger} \widetilde{b}_{n^{\prime}}+\frac{1}{2}\left(\mathcal{B}_{n n^{\prime}}(v(t)) \widetilde{b}_{n}^{\dagger} \widetilde{b}_{n^{\prime}}^{\dagger}-\mathcal{B}_{n n^{\prime}}^{*}(v(t)) \widetilde{b}_{n} \widetilde{b}_{n^{\prime}}\right)\right\}
$$

And finally

$$
\widetilde{H}=\frac{2 \pi v(t)}{l(t) \ln d} \sum_{n} n \widetilde{b}_{n}^{\dagger} \widetilde{b}_{n}+\mathcal{V}
$$

The representation (31) is very important because it clearly indicates that (i) the Hamiltonian for a field in a nonstationary cavity can be diagonalized if $\dot{v}=0$, and hence, we can use the notion of particles in a uniformly contracting cavity; and (ii) particle creation, as well as mode-to-mode scattering, takes place only when the boundary moves with acceleration. For our law of motion (9) these processes arise only in the initial $t=0$ and final $t=T$ moments of time. The fact that radiation processes are absent during the time when one boundary of a cavity moves with a constant velocity was first noticed by Fulling and Davis in Ref. (20]). 


\section{PARTICLE CREATION IN A UNIFORMLY CONTRACTING CAVITY}

We will now compute the number of particles created in a nonstationary cavity with the right mirror moving according to Eq. (9). We will note first that approach based on the Hamiltonian $\widetilde{H}$ is not convenient for this purpose. This is because velocity of the mirror in our approximation changes instantaneously, and thus $\widetilde{H}$ contains ill defined products of $\delta$ and $\theta$-functions. Thereagainst, the Hamiltonian $H^{\prime}$ (10) is not that singular even in our approximation (it contains only $\theta$-functions) and therefore operators $a_{n}(t)$ are continuous functions of time. This point plays the central role for our method of solution.

We start from the initial conditions

$$
\left.a_{n}(\chi)\right|_{\chi=-0}=a_{n}^{i n},
$$

where $a_{n}^{i n}$ are initial destruction operators which define the initial vacuum state by $a_{n}^{i n}|0\rangle_{i n}=0$. Due to continuity of operators $a_{n}(\chi)\left(a_{n}(-0)=a_{n}(+0)\right)$, we have the initial data for the operators $\tilde{b}_{n}(\chi)$,

$$
\tilde{b}_{n}(0)=\sum_{n^{\prime}}\left\{\alpha_{n n^{\prime}} a_{n^{\prime}}^{i n}+\beta_{n n^{\prime}} a_{n^{\prime}}^{i n \dagger}\right\} .
$$

According to Eq. (22) the operator $\widetilde{b}_{n}$ depends on $t$ (or $\chi$ ) by mean of phase rotation. Specifically, at $t=T$, we have:

$$
\tilde{b}_{n}\left(\chi_{f}\right)=e^{-2 \pi i n \Theta} \tilde{b}_{n}(0), \quad \Theta=\frac{\ln (1 / \rho)}{\ln d} .
$$

Here the quantity $\Theta$ measures the phase obtained by a particle in the principle mode $(n=1)$ during the squeezing period of the cavity. As it follows from Eq. (34), the phase factor is completely determined by the fractional part of $\Theta$. For further convenience, we will denote the fractional part of $\Theta$ by $\vartheta$,

$$
\vartheta=\operatorname{Frac}\left\{\frac{\ln (1 / \rho)}{\ln d}\right\} \text {. }
$$

The range of variation of the quantity $\vartheta$ is $0 \leq \vartheta<1$ (the symbol Frac denotes the fractional part of a number).

The operators $a_{n}\left(\chi_{f}-0\right)$, which are the solutions for Eqs. (12) with the initial conditions (32), can be now found from Eq. (26). Hence, again taking into account the continuity of operators $a_{n}$, we finally have for $a_{n}^{\text {out }}=$ $a_{n}\left(\chi_{f}+0\right)$ :

$$
a_{n}^{\text {out }}=\sum_{n^{\prime}}\left\{\alpha_{n^{\prime} n}^{*} \tilde{b}_{n^{\prime}}\left(\chi_{f}\right)-\beta_{n^{\prime} n} \tilde{b}_{n^{\prime}}^{\dagger}\left(\chi_{f}\right)\right\}
$$

By combining the three transformations (33), (34) and (36), we obtain:

$$
a_{n}^{\text {out }}=\sum_{n^{\prime}}\left\{U_{n n^{\prime}} a_{n^{\prime}}^{i n}+V_{n n^{\prime}} a_{n^{\prime}}^{i n \dagger}\right\}
$$

where the coefficients of the Bogolubov transformation (37) are given by

$$
\begin{aligned}
U_{n n^{\prime}} & =\sum_{n^{\prime \prime}}\left\{e^{-2 \pi i n^{\prime \prime} \vartheta} \alpha_{n^{\prime \prime} n}^{*} \alpha_{n^{\prime \prime} n^{\prime}}-e^{2 \pi i n^{\prime \prime} \vartheta} \beta_{n^{\prime \prime} n^{\prime}}^{*} \beta_{n^{\prime \prime} n}\right\}, \\
V_{n n^{\prime}} & =\sum_{n^{\prime \prime}}\left\{e^{-2 \pi i n^{\prime \prime} \vartheta} \alpha_{n^{\prime \prime} n}^{*} \beta_{n^{\prime \prime} n^{\prime}}-e^{2 \pi i n^{\prime \prime} \vartheta} \alpha_{n^{\prime \prime} n^{\prime}}^{*} \beta_{n^{\prime \prime} n}\right\} .
\end{aligned}
$$

Consider the expression for $V_{n n^{\prime}}$. Using Eq. (21), we get

$$
V_{n n^{\prime}}(d, \vartheta)=\frac{\sqrt{n n^{\prime}}}{4} \int_{-1}^{1} d y_{1} \int_{-1}^{1} d y_{2} \sum_{n^{\prime \prime} \geq 1} \frac{1}{n^{\prime \prime}}\left(\frac{1-v y_{1}}{1-v y_{2}}\right)^{2 \pi i n^{\prime \prime} / \ln d} \times\left\{e^{i \pi\left(n y_{1}+n^{\prime} y_{2}\right)-2 \pi i n^{\prime \prime} \vartheta}-e^{i \pi\left(n^{\prime} y_{1}+n y_{2}\right)+2 \pi i n^{\prime \prime} \vartheta}\right\} .
$$

After evaluation of the sum and double integral in (40) (see Appendix for details) we obtain

$$
V_{n n^{\prime}}=(-1)^{n+n^{\prime}} \frac{i(d-1) \sqrt{n n^{\prime}}}{2 \pi\left(n d^{\vartheta}+n^{\prime}\right)\left(n+n^{\prime} d^{1-\vartheta}\right)} \times\left\{e^{-2 \pi i n\left(d^{\vartheta}-1\right) /(d-1)}-e^{-2 \pi i n^{\prime}\left(d^{1-\vartheta}-1\right) /(d-1)}\right\} .
$$


The coefficient $U_{n n^{\prime}}$ differs from $V_{n n^{\prime}}$ by the only change $n^{\prime} \rightarrow-n^{\prime}$ everywhere, except for the common factor $\sqrt{n^{\prime}}$, so that

$$
U_{n n^{\prime}}=(-1)^{n+n^{\prime}} \frac{i(d-1) \sqrt{n n^{\prime}}}{2 \pi\left(n d^{\vartheta}-n^{\prime}\right)\left(n-n^{\prime} d^{1-\vartheta}\right)} \times\left\{e^{-2 \pi i n\left(d^{\vartheta}-1\right) /(d-1)}-e^{2 \pi i n^{\prime}\left(d^{1-\vartheta}-1\right) /(d-1)}\right\} .
$$

Finally, the energy (mode) distribution of created particles is given by

$$
\bar{N}_{n}(d, \vartheta)={ }_{i n}\left\langle 0\left|a_{n}^{\text {out }}{ }^{\dagger} a_{n}^{\text {out }}\right| 0\right\rangle_{\text {in }}=\sum_{n^{\prime} \geq 1}\left|V_{n n^{\prime}}\right|^{2}=\frac{(d-1)^{2} n}{\pi^{2}} \sum_{n^{\prime}=1}^{\infty} \frac{n^{\prime} \sin ^{2}\left[\frac{\pi\left(d^{\vartheta}-1\right)}{(d-1)}\left(n+n^{\prime} d^{1-\vartheta}\right)\right]}{\left(n d^{\vartheta}+n^{\prime}\right)^{2}\left(n+n^{\prime} d^{1-\vartheta}\right)^{2}} .
$$

One can see that the number of particles (43) created in each mode depends on two parameters. One of them is the Doppler factor $d$, which can be expressed either in terms of velocity of the moving boundary, $d=(1+v) /(1-v)$, or in terms of the corresponding Lorentz factor $\gamma=\left(1-v^{2}\right)^{-1 / 2}, d=\left(\gamma+\sqrt{\gamma^{2}-1}\right)^{2}$. The second parameter $\vartheta$ is determined by $d$ and a dimensionless squeeze rate of the cavity $\rho=l_{f} / l_{i}$ which, in turn, can be expressed in terms of the duration of squeezing $T$ as $\rho=1-v T / l_{i}$. Note that acceleration of the moving boundary,

$$
W=-v \delta(t)+v \delta(t-T)
$$

is determined by the same set of parameters. It is natural, since particles can be created by an accelerating boundary only.

An interesting feature of the obtained solution is that the number of created particles is a periodic function of the parameter $\log \rho$ with the period $\log d$. This can be traced already from the expansions (38), (39), and definition (35) of the parameter $\vartheta$. Surprisingly, the number of created particles completely vanishes if $\vartheta=0$, see Eq. (43). It is worth noting that $\bar{N}_{n}$ tends to zero also if $\vartheta \rightarrow 1$, so that the energy spectrum of created particles is a continuous function of parameter $\rho$. According to Eq. (35), $\vartheta$ turns into zero, and hence the number of created particles vanishes,

a)

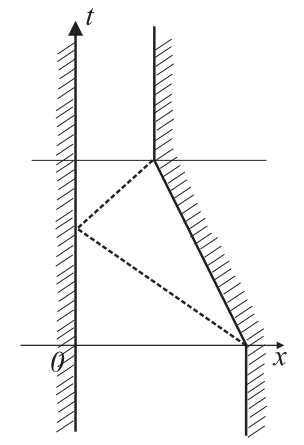

b)

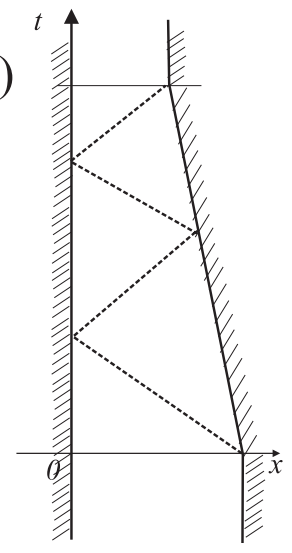

FIG. 1: A graphical illustration for null values of the number of created particles at a) $\rho=1 / d$, b) $\rho=1 / d^{2}$. The dashed lines represent world lines of a light ray, emitted by the moving boundary at $t=0$.

if $\rho=1 / d^{k}$, where $k=0,1,2, \ldots$. The value $k=0$ corresponds to a stationary case, while the others can be explained by destructive quantum interference between events of particle creation at the moments of acceleration $(t=0)$ and deceleration $(t=T)$ of the boundary. As it is illustrated by Fig. 1] the values of the squeeze rate $\rho=1 / d^{k}$ exactly correspond to the cases when a light ray, emitted by the right boundary at $t=0$, returns to it at $t=T$ after $k-1$ successive reflections and therefore can be absorbed at the moment of deceleration. Analogous arguments were employed by the authors of Ref. (20]) in discussion of the effect of vanishing of the renormalized energy flux in a uniformly expanding cavity, though only the case $k=1$ was considered in that work. It is worth mentioning that at $\rho=1 / d^{k}$ every level of the initial energy spectrum of the field inside the cavity converts in time interval $T$ to the value $\omega_{n}^{f}=\pi n / l_{i} \rho=\left(\pi n / l_{i}\right) d^{k}$ coinciding with the frequency acquired by a particle after $k-1$ successive reflections from the moving boundary, if this particle was emitted by the boundary moving with velocity $v$ at $t=0$.

The average number of particles, created in the principle $(n=1)$ and in the first excited $(n=2)$ modes as functions of $\vartheta$ are shown in Fig. 2 for several values of the moving boundary $\gamma$-factor. The same plots after the appropriate 


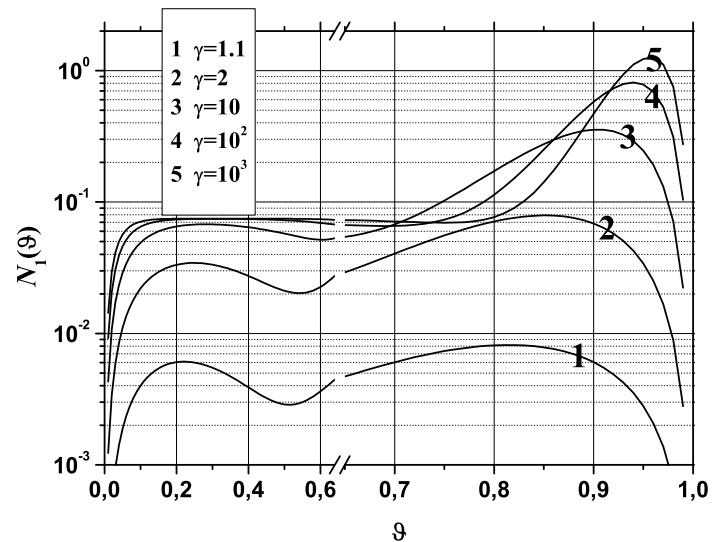

a)

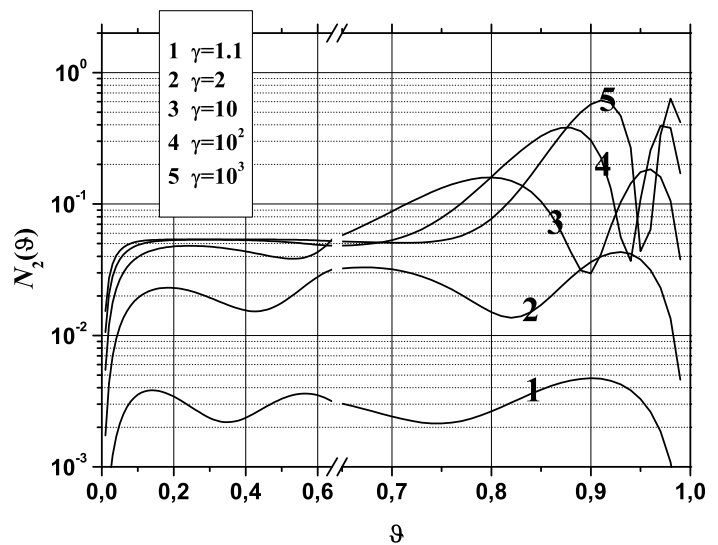

b)

FIG. 2: The average number of particles created in a) the principle mode $(n=1)$, and b) the first excited mode $(n=2)$ as a function of $\vartheta$ for different values of the moving boundary $\gamma$-factor.

scaling represent the first period of $\bar{N}_{n}$ dependence on $\log (1 / \rho)$.

One can see from Fig. [2] that at sufficiently large $\gamma$ plots for both $\bar{N}_{1}$ and $\bar{N}_{2}$ have a plateau at not too small $\vartheta$, and a strongly pronounced maxima (one for $n=1$ and two for $n=2$ ) at $\vartheta$ close to unity. It is seen also that the altitudes of the plateaus are growing with $\gamma$ remaining however rather small. To estimate the maximum possible altitude of a plateau we will note that in the limiting case $\gamma \rightarrow \infty(d \rightarrow \infty)$ at a fixed $\rho$ the instantaneous approximation considered in Ref. 13 becomes applicable. Indeed, one can easily make certain that Eq. (43) reduces to Eq. (6) of Ref. 13] under condition $d \gg n(1-\rho) / \rho^{1}$. For our estimation we need only high-energy tail of energy distribution in instantaneous approximation (see Eq. (10) of Ref. [13]) where we should put $\rho=0$ since not too small values of $\vartheta$ at $d \gg 1$ correspond to small $\rho$. Thus, for maximum values of $\bar{N}_{n}$ in the plateau region we have

$$
\bar{N}_{n}^{(p l)} \approx \frac{1}{2 \pi^{2} n}[\ln (2 \pi n)+C-1]
$$

where $C=0.5572$ is the Euler constant. For $n=1,2$ we have $\bar{N}_{1}^{(p l)} \approx 7.17 \cdot 10^{-2}$ and $\bar{N}_{2}^{(p l)} \approx 5.34 \cdot 10^{-2}$, respectively. So, in the plateau region the average number of created particles is small even for ultrarelativistic velocities of the moving boundary.

To estimate positions of the maxima and the peak values of $\bar{N}_{n}$ we should evaluate the sum in Eq. (43) under the conditions $d \gg 1,1-\vartheta \ll 1$. It is clear that the major contribution to the sum is given by $n_{e f f}^{\prime} \lesssim n d^{\vartheta}$. Thus we have:

$$
\bar{N}_{n} \approx \frac{n}{\pi^{2}} \sum_{n^{\prime}=1}^{\infty} \frac{n^{\prime} \sin ^{2}\left[\pi\left(n d^{\vartheta-1}-n^{\prime} d^{-\vartheta}\right)\right]}{\left(n+n^{\prime} d^{-\vartheta}\right)^{2}\left[n^{\prime}+n d^{\vartheta-1}\right]^{2}} \approx \frac{1}{\pi^{2} n} \sin ^{2}\left(\pi n d^{\vartheta-1}\right) \sum_{n^{\prime}=1}^{n d^{\vartheta}} \frac{n^{\prime}}{\left(n^{\prime}+n d^{\vartheta-1}\right)^{2}} .
$$

Since $n d^{\vartheta} \gg 1$, we can change summation over $n^{\prime}$ by integration. Finally, after evaluation of the integral we obtain in the leading logarithmic approximation:

$$
\bar{N}_{n} \approx \frac{1}{\pi^{2} n} \sin ^{2}\left(\pi n d^{\vartheta-1}\right) \ln (n d)
$$

We see from Eq. (45) that positions of the maxima are determined by the relations

$$
\pi n d^{\vartheta-1}=\pi(j+1 / 2), \quad j=0,1, \ldots(n-1) .
$$

\footnotetext{
${ }^{1}$ Note, that this condition of applicability of instantaneous approximation was obtained in Ref. 13] on the basis of merely qualitative arguments.
} 
This is an approximate condition but it becomes exact in the ultrarelativistic limit. For example, at $\gamma=2,10,10^{2}, 10^{3}$ for the single maximum position at $n=1$ we have respectively $\vartheta_{\max }=0.74,0.88,0.94,0.95$ from Eq. (46), and $\vartheta_{\max }=0.85,0.90,0.94,0.96$ as the result of computation according to exact formula (43).

The condition (46) can be reformulated in terms of wavelengths as

$$
(2 j+1) \lambda_{n}^{f} / 2=2 l_{i} / d^{k+1},
$$

where $\lambda_{n}^{f}=2 l_{f} / n$ is the wavelength corresponding to the $n$-th harmonic in the final state. The physical meaning of this condition can be clarified by the following qualitative consideration.

We have shown, see Eqs. (31), (30), that particles are created inside the cavity only at the moments of start $(t=0)$ and stopping $(t=T)$ of its boundaries. Physically, it is clear that in the framework of the applied approximation (instantaneous acceleration) the created particles constitute an extremely narrow wave packet (cluster) which propagates then inside the cavity without spreading successively colliding with its boundaries, see Fig. [3 Such picture agrees with the results of calculation of the energy momentum tensor performed by Fulling and Davies in Ref. (20]).

Let us describe the clusters of particles created at the moments $t=0$ and $t=T$ by classical fields $\Phi_{1}(x, t)$ and $\Phi_{2}(x, t)$ respectively. The function $\Phi_{2}(x, T)$ describes the second cluster produced by the boundary stopping at the moment $t=T$. It is located exactly at the point $x=l_{f}$. The first cluster at the same moment of time is located at some distance $\Delta$ left to the first one. The form of the cluster $\Phi_{1}$ is absolutely the same and differs from $\Phi_{2}$ only by sign since the boundary accelerations at two moments of particle creation were of opposite sign. Thus, $\Phi_{1}(x, T)=-\Phi_{2}(x-\Delta, T)$. Expanding the fields $\Phi_{i}$ in terms of stationary out-modes

$$
\psi_{n}^{\text {out }}(x, t)=\frac{1}{\sqrt{\pi n}} \sin \left(\frac{\pi n}{l_{f}} x\right) e^{-i \omega_{f n} t}, \quad \omega_{f n}=\left(\pi / l_{f}\right) n,
$$

for the total field $\Phi(x, T)=\Phi_{1}(x, T)+\Phi_{2}(x, T)$ we obtain

$$
\Phi(x, T)=\sum_{n=1}^{\infty} \frac{2}{\sqrt{\pi n}}\left(c_{n} e^{-i \omega_{f n} T}+c_{n}^{*} e^{i \omega_{f n} T}\right) \cos \left(\frac{\pi n}{l_{f}}\left(x-\frac{\Delta}{2}\right)\right) \sin \left(\frac{\pi n}{2 l_{f}} \Delta\right) .
$$

Thus, we see that contribution of the $n$-th mode to the field $\Phi(x, T)$ is absent if $n \Delta / 2 l_{f}=j, j=0,1,2, \ldots$ (destructive interference of two clusters), or it is maximal if

$$
n \Delta / 2 l_{f}=j+1 / 2, \quad j=0,1,2, \ldots
$$

(constructive interference). In view of the restriction $\Delta \leq l_{f}$, we have $2 j+1 \leq n$. Since particle creation and mode interaction are absent in a stationary cavity, these conditions hold at arbitrary moment of time $t>T$. Now, the question is whether it is possible to match Eq. (48) with conditions (47) (as well as the corresponding conditions for minima).

Assume that the first cluster undergoes $k$ collisions with the moving boundary during the period of contraction $T$. It can be easily found that the last collision takes place at the point $x_{k}=l_{i} / d^{k}$ at the moment of time $t_{k}=\left(l_{i} / v\right)\left(1-1 / d^{k}\right)$. During the period of time $T-t_{k}$ the cluster either collides with the boundary at rest, if $T-t_{k}>x_{k}$ (Fig. Ba $)$, or not, if $T-t_{k}<x_{k}$ (Fig. [3b). The quantity $\Delta$ for these cases is given by the following relations respectively:

$$
\Delta=l_{f} \pm\left[x_{k}-\left(T-t_{k}\right)\right] .
$$

Then Eq. (48) is equivalent to two relations

$$
\frac{l_{i}}{d^{k}} \frac{1-v}{v}=\left\{\begin{array}{ll}
\frac{2 l_{f}}{n}\left(n \frac{1+v}{2 v}-j-\frac{1}{2}\right), & T-t_{k}>x_{k} \\
\frac{2 l_{f}}{n}\left(j+\frac{1}{2}+n \frac{1-v}{v}\right), & T-t_{k}<x_{k}
\end{array},\right.
$$

where $j \leq(n-1) / 2$. In the ultrarelativistic limit $1-v \ll 1(d=2 /(1-v))$ the conditions (49) take on form of Eq. (47) with $0 \leq j \leq n-1$. Our analysis shows that physically the conditions (47) mean that maxima of $N_{n}$ appear in the cases when the first cluster is located at a distance equal to an odd number of half-waves $\lambda_{n}^{f} / 2$ from the point of creation of the second cluster. It is worth emphasizing that kinematics of our problem permits implementation of this condition only in the ultrarelativistic case. Therefore the less is the speed of the boundary $v$, the less pronounced are the maxima and $N_{n} \neq 0$ in minima, compare Fig. [2]

The values of $\widetilde{N}_{n}$ in the points of maxima are given by Eq. (45) with logarithmic accuracy, and thus cannot reproduce numerical results just as well as their positions. However, Eq. (45) reproduces the main features of spectra nearby the 
a)

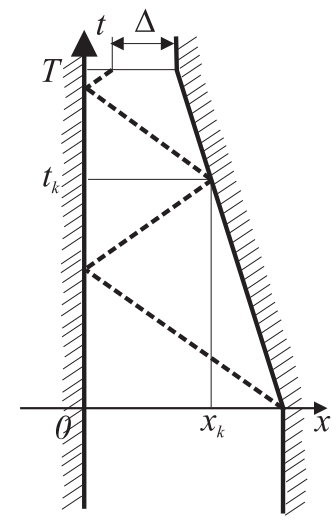

b)

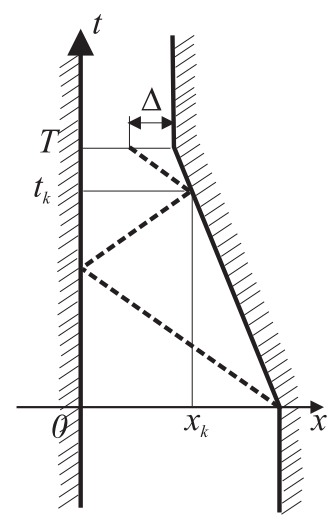

FIG. 3: World lines of cavity boundaries (solid lines) and of the cluster of particles (dashed line) created at $t=0$ for a) $T-t_{k}>x_{k}$, and b) $T-t_{k}<x_{k}$. The number of collisions with the moving boundary $k=1$.

maxima qualitatively correctly. It is important that the height of the maxima grows as $\log d$, or equivalently, as log $\gamma$ in the ultrarelativistic limit due to strong interactions between the modes. Clearly, the effect of DCE amplification arises due to constructive interference of events of particle creation at $t=0$ and $t=T$. For example, if $\gamma=10^{3}$, then for certain values of $\rho$ (or $T$ ) in the mean more than one particle in the principle mode (or a little bit less than one particle in the first excited mode) can be created.

\section{DISCUSSION}

An exact solution to the problem of dynamical Casimir effect in a one-dimensional uniformly contracting cavity was found. This became possible due to existence of stable non-interacting particle states in such a cavity. As a consequence, we succeeded in diagonalization of the Hamiltonian for a many-mode quantum field in a cavity with uniformly moving boundaries (a generalization to the case with both boundaries moving uniformly is straightforward, but rather cumbersome). The developed method opens us possibilities to solve exactly many other one-dimensional DCE problems for cavities with boundaries moving along polygon-like world lines, including the interesting case of vibrating boundaries. A new representation for the field Hamiltonian was proposed in one-dimensional problem. It was demonstrated explicitly that particle creation as well, as mode-to-mode interaction is absent in a nonstationary cavity if its boundaries are moving uniformly. However, the same approach can not be applied to physically more realistic three-dimensional problem, although a complete set of classical solutions generalizing Eq. (16) is known for this case as well [9]. This is due to breakdown of stability of particle states and presence of interactions between them in three-dimensional problem.

It was shown that the number of particles, created in each mode, depends periodically on the logarithm of the squeezing rate of the cavity $\rho$, which is equal to the ratio of the final and initial sizes of the cavity and is related to duration of contracting in a rather simple way. This dependence is not monotonous. For the given velocity of the moving boundary, there exists an infinite sequence of values $\rho_{k}$ for which particles are not produced. On the other hand, it is possible to specify an infinite set of combinations of parameters, which correspond to an optimal regime of particle production. Theoretically, at sufficiently large Lorentz factor $\gamma$ for the moving boundary, the number of created particles can be done arbitrary large. It is worth noting, however, that the mentioned values of Lorentz factors are indeed very large. For example, the value $\gamma \sim 10^{3}$ for production of a single particle is necessary. We should also mention that the number of created particles in the case of a cavity with ultrarelativistic boundaries depends on $\gamma$ rather slowly (proportional to $\log \gamma$ ).

The non-monotonous character of dependence of the number of created particles on $\rho$ is also present in the framework of an oversimplified "one-mode" model. However, correct estimations, especially near the maxima of particle production rate, and even correct qualitative understanding require taking into account mode-to-mode interactions. This is true in principle at consideration of any non-perturbative feature of DCE. Therefore, it is very important to consider exactly soluble models. It should be noticed, that the approximation of uniform motion of the boundaries, separated by moments of instant infinite accelerations, does not work well for high-frequency modes. For example, the energy spectrum (43) has an inadmissibly slowly decreasing tail $\left(\bar{N}_{n} \propto 1 / n\right)$ leading to an infinite total number of 
created particles. These problems can be resolved by smoothing instant infinite accelerations, but the corresponding problem ceases be exactly soluble.

\section{Acknowledgments}

We thank M.I. Gozman, V.D. Mur, V.S. Popov and participants of the Fourth D.N. Klyshko seminar for discussion of the results and helpful remarks. This work was supported by the Russian Foundation for Basic Research (grant 06-02-17370-a), by the Ministry of Science and Education of Russian Federation (grant RNP 2.11.1972), and by the Russian Federation President grants MK-2279.2005.2 and NSh-320.2006.2.

\section{APPENDIX A: EVALUATION OF THE INTEGRAL IN EQ. (40)}

The coefficient $V_{n n^{\prime}}$ (40) is represented by a difference of two integrals. After the change of integration variables $y_{1} \leftrightarrow y_{2}$ in the second of them, $V_{n n^{\prime}}$ reduces to the form:

$$
V_{n n^{\prime}}=\frac{i \sqrt{n n^{\prime}}}{2} \int_{-1}^{1} d y_{1} \int_{-1}^{1} d y_{2} e^{i \pi\left(n y_{1}+n^{\prime} y_{2}\right)} \operatorname{Im} \sum_{n^{\prime \prime} \geq 1} \frac{e^{2 \pi i n^{\prime \prime} Z\left(y_{1}, y_{2}\right)}}{n^{\prime \prime}}
$$

where

$$
Z\left(y_{1}, y_{2}\right)=\log _{d}\left(d^{-\vartheta} \frac{1-v y_{1}}{1-v y_{2}}\right)
$$

For calculation of the sum in Eq. A1 we will use formula 1.441.1 from 21] generalized for arbitrary values of $Z$

$$
\operatorname{Im} \sum_{n^{\prime \prime} \geq 1} \frac{e^{2 \pi i n^{\prime \prime} Z}}{n^{\prime \prime}}=\pi\left(\frac{1}{2}-Z\right)+\pi \sum_{k=-\infty}^{\infty} k \theta(Z-k) \theta(k+1-Z) .
$$

Obviously, the first term in the RHS of (A3) gives zero contribution to the integral (A1). Taking into account that $-1-\vartheta \leq Z\left(y_{1}, y_{2}\right) \leq 1-\vartheta$ inside the integration domain, and $0 \leq \vartheta<1$ by definition, we obtain

$$
\begin{array}{r}
V_{n n^{\prime}}=-\frac{i \pi \sqrt{n n^{\prime}}}{2} \int_{-1}^{1} d y_{1} \int_{-1}^{1} d y_{2} e^{i \pi\left(n y_{1}+n^{\prime} y_{2}\right)}\{\theta(-Z) \theta(Z+1) \\
+2 \theta(-Z-1) \theta(Z+2)\} .
\end{array}
$$

It is clear that the combination of $\theta$-functions

$$
\theta(Z)+\theta(-Z) \theta(Z+1)+\theta(-Z-1) \theta(Z+2)
$$

is equivalent to 1 in the domain of integration, and thus

$$
\iint d^{2} y e^{i \pi\left(n y_{1}+n^{\prime} y_{2}\right)}\{\theta(Z)+\theta(-Z) \theta(Z+1)+\theta(-Z-1) \theta(Z+2)\}=0 .
$$

Therefore, we have:

$$
V_{n n^{\prime}}=\frac{i \pi \sqrt{n n^{\prime}}}{2} \iint d^{2} y e^{i \pi\left(n y_{1}+n^{\prime} y_{2}\right)}\{\theta(Z)-\theta(-Z-1)\} .
$$

The integral A5 can be easily reduced to the form

$$
V_{n n^{\prime}}=\frac{i \pi \sqrt{n n^{\prime}}}{2}\left\{\int_{-1}^{a} d y_{1} \int_{b\left(y_{1}\right)}^{1} d y_{2}-\int_{a}^{1} d y_{1} \int_{-1}^{c\left(y_{1}\right)} d y_{2}\right\} e^{i \pi\left(n y_{1}+n^{\prime} y_{2}\right)},
$$


where $a=1-2\left(d^{\vartheta}-1\right) /(d-1), b\left(y_{1}\right)=\left(1-d^{-\vartheta}\right) / v+d^{-\vartheta} y_{1}$, and $c\left(y_{1}\right)=\left(1-d^{1-\vartheta}\right) / v+d^{1-\vartheta} y_{1}$. After simple calculations we now obtain the expression (41) for $V_{n, n^{\prime}}$.

[1] Moore G T 1970 J. Math. Phys 112679.

[2] Dodonov V V 1995, Phys. Lett. A 207 126; Dodonov V V, Klimov A B 1996, Phys. Rev. A 532664.

[3] Dodonov V V and Klimov A B 1996 Phys. Rev. A 532664.

[4] Dodonov V V, Man'ko V I and Man'ko O V 1991 Proceedings of Lebedev Physics Institute vol 200 (Moscow: Nauka) p 155.

[5] Jaekel M T and Reynaud S 1992. J. Phys. I (France) 2149.

[6] Law C K 1994 Phys. Rev. A 49 433, 1995 Phys. Rev. A 512537.

[7] Dodonov V V 1995 Phys. Lett. A 207126.

[8] Klimov A B, Altuzar V 1997 Phys. Lett. A 22641.

[9] Dodonov V V 2001 Advances in Chemical Physics vol 119 (John Wiley \& Sons, Inc) p 309.

[10] Yablonovitch E 1989 Phys. Rev. Lett. 621742.

[11] Lozovik Yu E, Tsvetus V G and Vinogradov E A 1995 Phys. Scr. 52284.

[12] Dodonov A V, Dodonov E V and Dodonov V V 2003, Phys. Lett. A 317378.

[13] Fedotov A, Narozhny N and Lozovik Yu 2005 J. Opt. B: Quantum Semiclass. Opt. B 7 S64.

[14] M. Bordag, G. Petrov and D. Robaschik, Yad. Fiz. 39, 1315 (1984) [Sov. J. Nucl. Phys. 39, 828 (1984)].

[15] M. Bordag, F.-M. Dittes and D. Robaschik, Yad. Fiz. 43, 1606 (1986) [Sov. J. Nucl. Phys. 43, 1034 (1986)].

[16] Razavy M, and Terning J 1985 Phys. Rev. D 31307.

[17] L. D. Landau and E. M. Lifshitz, Mechanics (3rd Edition, Butterworth-Heinemann, 1981).

[18] Yu.E. Lozovik, N.B. Narozhny and A.M. Fedotov, Proceedings of the International Conference I. Ya. Pomeranchuk and Physics at the Turn of Centuries, Edited by A. Berkov, N. Narozhny and L. Okun, (World Scientific, Singapore, 2004), p.446.

[19] N.D. Birrell and P.C.W. Davies, Quantum Fields in Curved Space (Cambridge University Press, Cambridge, 1982).

[20] Fulling S A and Davies P C W 1975, Proc. R. Soc. Lond.A 348393.

[21] I.S. Gradshteyn, and I.M. Ryzhik, Tables of Integrals, Series, and Products (Academic, New York, 1967). 